INPLASY

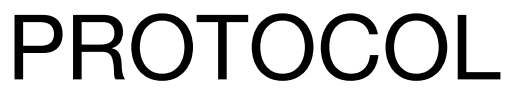

To cite: Han. Risk factors and prevalence of DVT after total hip replacement $A$ protocol for meta-analysis. Inplasy protocol 2020110112. doi:

10.37766/inplasy2020.11.0112

Received: 25 November 2020

Published: 26 November 2020

Corresponding author:

Zhe Han

hanzhe1989@yeah.net

Author Affiliation:

Tianjin hospital

Support: No.

Review Stage at time of this submission: Data analysis.

Conflicts of interest: No.

\section{Risk factors and prevalence of DVT after total hip replacement A protocol for meta-analysis}

Han, Z1.

\section{INTRODUCTION}

Review question / Objective: The risk factors of thrombosis after hip arthroplasty are numerous and complex. It can enable patients to progress step by step from asymptomatic, eventually leading to death. However, it is still difficult to effectively prevent damage caused by it. Therefore, it is necessary to further study the risk factors and effective preventive measures of DVT. To provide appropriate intervention measures for high-risk factors to prevent the occurrence and development of DVT and to control it before irreversible damage.

Condition being studied: The common hip joint diseases are severe osteoarthritis, rheumatoid arthritis, advanced aseptic 
necrosis of the femoral head and femoral neck fracture. At present, one of the effective methods for the treatment of the above-mentioned hip diseases is hip arthroplasty. Artificial hip arthroplasty has a history of 30 years. It is widely used in clinic because of its mature technology, good effect, postoperative pain relief and complete recovery of lower limb function. This technique has remarkable clinical effect in helping patients recover hip joint function and relieving pain, and has been widely recognized by medical workers and patients. although this method can better restore the functional activities of patients' lower limbs, eliminate pain and improve their quality of life, it will also bring some complications after operation. Such as infection, periprosthetic fracture, dislocation, lower limb unequal length, lower limb deep venous thrombosis and other problems, of which lower limb deep venous thrombosis (DVT) is the most common. Related studies have shown that if no preventive measures are taken, the incidence of postoperative DVT is $34.1 \%$ to $77.2 \%$, the incidence of symptomatic pulmonary embolism (PE,) is in the range of $1.9 \%$ to $6.8 \%$, and the mortality is as high as $2 \%$. DVT

\section{METHODS}

Participant or population: Types of participants. formation of DVT after total hip replacement (THA).

Intervention: None.

\section{Comparator: None.}

Study designs to be included: Types of studies. Inclusion: (1) Randomized controlled trial; (2) cohort studies; (3) casecontrol studies. Exclusion: (1) Non-Chinese and English literature; (2)Incomplete or missing research data; (3) Unable to obtain original documents; (4) Repeated publication of literature; (5)Editorials (6) Commentaries.

Eligibility criteria: Types of studies. Inclusion: (1) Randomized controlled trial; (2) cohort studies; (3) case-control studies.
Exclusion: (1) Non-Chinese and English literature; (2)Incomplete or missing research data; (3) Unable to obtain original documents; (4) Repeated publication of literature; (5)Editorials (6) Commentaries

Information sources: Electronic searches strategy. "DVT after total hip replacement" was used as the English search term, database retrieval was carried out on MEDLINE, Embase, ovid, Web of Science, Wanfang, CNKI database, and literatures on DVT after total hip replacement published from the establishment of the database to November $\mathbf{2 0 2 0}$ were collected systematically.

Main outcome(s): Risk factors. advanced age, male gender, Basic disease, operation time, postoperative immobilization time, international standardized ratio of prothrombin time, CDFI value of superficial femoral vein.

Quality assessment / Risk of bias analysis: Two reviewers will independently assess the quality of included studies by using the Newcastle-Ottawa Scale (NOS) for nonrandomized studies. This is a specific method for assessing the quality of cohort and case-control study. There are 8 entries in 3 modules, among which 4 points are selected for study population, 2 points for comparability between groups, and 3 points for measurement of results. The total score $\geq 6$ points is considered as highquality research literature. The Cochrane bias risk assessment tool was used to evaluate the final included RCTs: random allocation method; allocation plan concealment; blinding of research subjects and experimenters; blinding of outcome evaluators; completeness of result data; selective reporting of studies Results; other sources of bias, including potential bias related to the specific research design of the study. For each of the above items, make a judgment of"low risk of bias", "high risk of bias" and "uncertain risk of bias". Disagreement will be solved by discussion or by consulting the third person.

Strategy of data synthesis: Statistical analysis was performed on the extracted 
data using Stata 12.0 software. For measurement data, the weighted mean difference (WMD) is used as the combined effect size; for binary variable data, the odds ratio (OR) is used as the combined effect size. Use the statistics 12and $P$ values to test the heterogeneity of the combined literature. If $P \geq 0.1, I 2<50 \%$, it indicates that there is homogeneity among the studies or the heterogeneity is within the acceptable range, and the fixed effects model is used to merge the calculation of the effect size; on the contrary, it is considered that there is heterogeneity between the studies. Egger's method and beggs method were used to assess publication bias.

Subgroup analysis: If the evidence is sufficient, we will conduct a subgroup analysis to determine the difference between different gender, age etc.

Sensibility analysis: None.

Country(ies) involved: multi-national collaborations.

Keywords: Risk factors, DVT, THA.

Contributions of each author:

Author 1 - Zhe Han - Author 1 drafted the manuscript. 